The Astrophysical Journal Supplement Series, 154:166-169, 2004 September

(C) 2004. The American Astronomical Society. All rights reserved. Printed in U.S.A.

\title{
OBSCURED AND UNOBSCURED ACTIVE GALACTIC NUCLEI IN THE SPITZER SPACE TELESCOPE FIRST LOOK SURVEY
}

\author{
M. Lacy, ${ }^{1}$ L. J. Storrie-Lombardi, ${ }^{1}$ A. Sajina, ${ }^{2}$ P. N. Appleton, ${ }^{1}$ L. Armus, ${ }^{1}$ S. C. Chapman, ${ }^{1}$ P. I. Choi, ${ }^{1}$ \\ D. Fadda, ${ }^{1}$ F. Fang, ${ }^{1}$ D. T. Frayer, ${ }^{1}$ I. Heinrichien, ${ }^{1}$ G. Helou,${ }^{1}$ M. Im, ${ }^{3}$ F. R. Marleau, ${ }^{1}$ F. Masci, ${ }^{1}$ \\ D. L. Shupe, ${ }^{1}$ B. T. Soifer, ${ }^{1}$ J. Surace, ${ }^{1}$ H. I. Teplitz, ${ }^{1}$ G. Wilson, ${ }^{1}$ and L. Yan ${ }^{1}$ \\ Received 2004 March 26; accepted 2004 April 28
}

\begin{abstract}
Selection of active galactic nuclei (AGNs) in the infrared facilitates the discovery of AGNs whose optical emission is extinguished by dust. In this paper, we use the Spitzer Space Telescope First Look Survey (FLS) to assess the fraction of AGNs with mid-infrared (MIR) luminosities that are comparable to quasars and that are missed in optical quasar surveys because of dust obscuration. We begin by using the Sloan Digital Sky Survey (SDSS) database to identify 54 quasars within the $4 \mathrm{deg}^{2}$ extragalactic FLS. These quasars occupy a distinct region in MIR color space by virtue of their strong, red continua. This has allowed us to define an MIR color criterion for selecting AGN candidates. About 2000 FLS objects have colors that are consistent with them being AGNs, but most are much fainter in the MIR than the SDSS quasars, which typically have $8 \mu \mathrm{m}$ flux densities $S_{8.0} \sim 1 \mathrm{mJy}$. We have investigated the properties of 43 objects with $S_{8.0} \geq 1 \mathrm{mJy}$ that satisfy our AGN color selection. This sample should contain both unobscured quasars as well as AGNs that are absent from the SDSS survey because of extinction in the optical. After removing 16 known quasars, three probable normal quasars, and eight spurious or confused objects from the initial sample of 43, we are left with 16 objects that are likely to be obscured quasars or luminous Seyfert 2 galaxies. This suggests that the numbers of obscured and unobscured AGNs are similar in samples selected in the MIR at $S_{8.0} \sim 1 \mathrm{mJy}$.
\end{abstract}

Subject headings: galaxies: Seyfert — infrared: galaxies — quasars: general

\section{INTRODUCTION}

The number of active galactic nuclei (AGNs) missed in optical or soft X-ray surveys because of obscuring columns of dust and gas in the host is still an open question (e.g., Webster et al. 1995; Cutri et al. 2001; Gregg et al. 2002; Norman et al. 2002; Richards et al. 2003). Understanding and quantifying the population of obscured AGNs is important if we are to obtain a complete picture of the buildup of black holes in the nuclei of galaxies. The close link between bulge mass and black hole mass (e.g., Magorrian et al. 1998) implies that understanding this history will also help us to understand the galaxy formation process as a whole.

The local mass density in black holes is dominated by the $\sim 10^{8.5} M_{\odot}$ black holes in $L_{*}$ elliptical galaxies. When accreting at Eddington rates, these correspond to luminous quasars $\left(M_{B} \approx-25\right)$. Recent work on the X-ray background, however, indicates that most of the mass buildup may occur on longer timescales at sub-Eddington rates, mostly in obscured AGNs (Cowie et al. 2003). Besides X-ray searches, other techniques for finding obscured AGNs have also been employed. A sample of luminous narrow-line AGNs has been selected from the Sloan Digital Sky Survey (SDSS; Zakamska et al. 2003). Quasar samples selected in the near-infrared from the Two Micron All-Sky Survey (2MASS; Cutri et al. 2001; Glikman et al. 2004) have identified a number of quasars with sig-

\footnotetext{
${ }^{1}$ Spitzer Science Center, California Institute of Technology, MC 220-6, Pasadena, CA 91125; mlacy@ipac.caltech.edu, lisa@ipac.caltech.edu.

2 Department of Physics and Astronomy, University of British Columbia, 6224 Agricultural Road, Vancouver, BC V6T 1Z1, Canada; sajina@astro .ubc.ca.

3 Astronomy Program, School of Earth and Environmental Sciences, Seoul National University, Shillim-dong, Kwanak-gu, Seoul 2-880-9010, South Korea.
}

nificant optical extinction and X-ray columns. Even in the mid-infrared (MIR), a highly obscured AGN will still have significant extinction. However, the disk- or torus-like geometry of the obscuring material means that even if the extinction to the broad-line region is high, MIR light will usually be visible unless the geometry is truly edge-on or the AGN is completely enveloped in a dense cloud of dust and gas (Pier \& Krolik 1993; Efstathiou \& Rowan-Robinson 1995). Thus, an MIR survey can usefully address the question of the number of obscured AGNs.

Studies with the Infrared Space Observatory (ISO) have shown that the strong MIR continuum associated with AGNs provide a unique spectral signature that can be used to distinguish AGNs from starbursts. The MIR continuum from galaxies arises mostly from three sources: $\mathrm{H}$ II regions dominated by emission from very small dust grains (producing a steeply rising continuum at $12-16 \mu \mathrm{m}$ ), photodissociation regions dominated by bands of polycyclic aromatic hydrocarbon (PAH) emission, and AGNs dominated by a strong 3-10 $\mu \mathrm{m}$ continuum. Laurent et al. (2000) show that the spectral energy distributions (SEDs) arising from each of these is sufficiently distinct to allow discrimination of AGNs from star-forming galaxies based on their MIR SEDs. Using these ideas, Haas et al. (2004) combined near- and mid-infrared colors to select AGNs from a parallel ISO survey. With the advent of Spitzer (Werner et al. 2004), it has become possible to obtain MIR photometry for large samples of field galaxies, and thus to use the MIR SEDs to identify specific populations of AGNs and star-forming galaxies. In this paper we use SDSS quasars to provide an empirical localization of the AGN population in MIR color space. We then select a sample of candidate obscured AGNs with $8 \mu \mathrm{m}$ flux densities $S_{8.0} \geq 1 \mathrm{mJy}$ and compare their optical identifications and estimated redshifts with those of the SDSS quasars with $S_{8.0} \geq 1 \mathrm{mJy}$. We use the 
main field of the extragalactic component of the Spitzer First Look Survey (FLS), a shallow, $4 \mathrm{deg}^{2}$ survey with the Infrared Array Camera (IRAC; Fazio et al. 2004) and the Multiband Imaging Photometer for Spitzer (MIPS; Rieke et al. 2004), for which an extensive multiwavelength ancillary data set exists. We assume a cosmology with $\Omega_{M}=0.3, \Omega_{\Lambda}=0.7$, and $H_{0}=70 \mathrm{~km} \mathrm{~s}^{-1} \mathrm{Mpc}^{-1}$.

\section{THE SPITZER FLS MAIN-FIELD DATA SET}

The FLS observations were made in 2003 December (Program ID 26, Spitzer AOR keys 0003861504, 0003861760 , 0003862016， 0003862016， 0003862272，0003862528, 0003862784, 0003863040, 0003863296, 0003863552). We have used preliminary versions of the IRAC catalog (M. Lacy et al. 2004, in preparation) and the MIPS $24 \mu \mathrm{m}$ catalog (D. Fadda et al. 2004, in preparation). The IRAC catalog has flux-density limits ( $5 \sigma$ in a $5^{\prime \prime}$ diameter aperture) in the four IRAC channels with nominal central wavelengths of 3.6, 4.5, 5.8 , and $8.0 \mu \mathrm{m}$ of $S_{3.6} \approx 7 \mu \mathrm{Jy}, S_{4.5} \approx 8 \mu \mathrm{Jy}, S_{5.8} \approx 60 \mu \mathrm{Jy}$, and $S_{8.0} \approx 50 \mu \mathrm{Jy}$, respectively. The MIPS $24 \mu \mathrm{m}$ catalog has a flux density limit of $\sim 300 \mu \mathrm{Jy}$.

\section{THE SDSS QUASARS}

The Sloan Data Release 1 (DR1) quasar survey (Schneider et al. 2003) contains 54 quasars that fall within the main field of the FLS. All were detected with IRAC, and all but one were seen at $24 \mu \mathrm{m}$ with MIPS. Three more quasars were found as a result of spectroscopic follow-up of the radio survey by Condon et al. (2003) using the Kast spectrograph at Lick Observatory.

\section{THE POSITION OF SDSS QUASARS IN MIR COLOR-COLOR PLOTS}

An IRAC color-color plot using all four broadband channels of the IRAC instrument is shown in Figure 1. The dots indicate the location of $\sim 16,000$ objects in the main-field catalog. Plotting the $8.0 \mu \mathrm{m} / 4.5 \mu \mathrm{m}$ ratio against the $5.8 \mu \mathrm{m} / 3.6 \mu \mathrm{m}$ ratio makes the color-color plot effective for separating objects with blue continua from those with red. Most objects have blue colors in both axes. These are most likely stars or low-redshift galaxies whose SEDs are weak in nonstellar light (e.g., elliptical galaxies). From this clump, two distinct "sequences" can be seen. One has blue colors in $S_{5.8} / S_{3.6}$ and very red colors in $S_{8.0} / S_{4.5}$. These are most likely low-redshift $(z \lesssim 0.2)$ galaxies with the centers of their 6.2 and $7.7 \mu \mathrm{m}$ PAH emission bands (Puget \& Léger 1989) redshifted into the IRAC $8.0 \mu \mathrm{m}$ filter. The other sequence has red colors in both pairs of filters, and it is on this sequence that the SDSS and radio-selected quasars lie. Note that strong stellar light will shift the $S_{5.8} / S_{3.6}$ ratio to the blue, as illustrated by the two filled squares in Figure 1 . These objects are classed as quasars in the SDSS database, but have resolved host galaxies in the IRAC images, and so are classed as Seyfert 1's in this study.

\section{MIR SELECTION OF CANDIDATE AGNS}

We used Spitzer colors of the SDSS quasars to empirically define a color selection that would pick out objects with MIR SEDs that are consistent with their being AGNs. This is the region enclosed by the dashed line in Figure 1. Our modeling of MIR SEDs based on ISO spectra (A. Sajina et al. 2004, in preparation) suggests that most of the objects in this region are indeed dominated by AGN emission, although we expect some

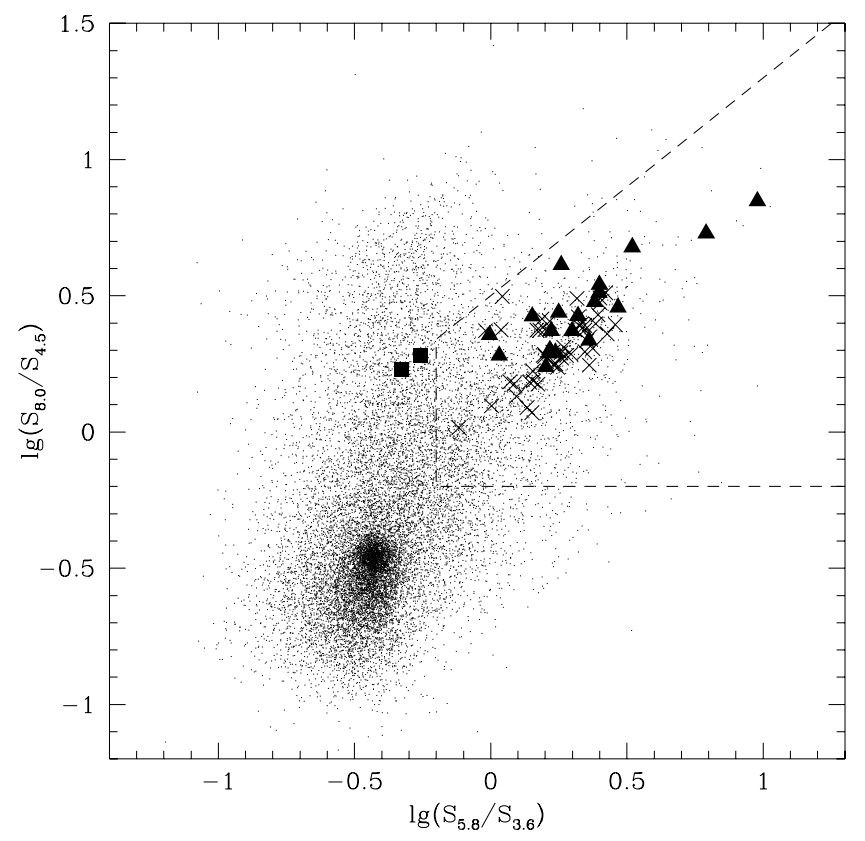

FIG. 1.-IRAC color-color plot using the main-field FLS data. Dots represent the $\sim 16,000$ objects with "clean" detections in all four IRAC bands. Crosses indicate the colors of all the SDSS and radio-selected quasars, squares the SDSS Seyfert 1 galaxies, and triangles the bright $\left(S_{8.0}>1 \mathrm{mJy}\right)$ sample of obscured AGNs. The dashed line shows the color criteria used to pick out the AGN sample.

contamination by star-forming galaxies close to the boundaries. There are $\sim 2000$ sources in this region that are therefore likely to contain AGNs. Most of them are fainter in the MIR than the SDSS quasars, which are typically much brighter than the FLS flux density limits.

To pick a sample of AGNs selected in the MIR that we could directly compare with the SDSS quasar sample, we examined the distribution of $S_{8.0}$ for the SDSS sample $(8.0 \mu \mathrm{m}$ is the longest IRAC wavelength and is therefore least affected by dust obscuration). This distribution showed a peak at $S_{8.0} \approx$ $1 \mathrm{mJy}$, and we therefore assumed that at $S_{8.0} \geq 1 \mathrm{mJy}$, most of our MIR-selected AGNs would appear in the SDSS sample if they were not obscured by dust.

There are 43 objects within the AGN selection region of the color-color plot that have $S_{8.0} \geq 1 \mathrm{mJy}$ and "clean" detections in the IRAC FLS catalog (i.e., unblended [about $80 \%$ of the four-band detections], and outside the halos of bright stars and unaffected by multiplexer bleed or pulldown $[97.6 \%$ of the survey area]). The steps used to determine which of these were candidate obscured AGNs were as follows: (1) we matched the sample of 43 bright AGN candidates to the SDSS quasar list, finding that 14 of our 43 objects are known SDSS quasars; (2) we next matched to our list of radio-selected quasars, finding two objects in common; (3) we examined the objects by eye on the FLS mosaics and rejected seven more objects from the sample on the basis of their being either saturated stars or confused in the IRAC images. This left 20 objects in the sample, which are our bright, obscured AGN candidates. These are shown as triangles in Figure 1 and are listed in Table 1. As a check on the AGN nature of these candidates, in Figure 2 we plot a color-color plot that extends the wavelength range to MIPS's $24 \mu \mathrm{m}$. The large wavelength difference between 8 and $24 \mu \mathrm{m}$ makes the interpretation of the MIPS/IRAC colorcolor plots more complicated, but it can be seen that most of the obscured AGN candidates lie in the same region of the 
TABLE 1

Candidate AGNs not in SDSS DR1 with $S_{8.0} \geq 1 \mathrm{mJy}$

\begin{tabular}{|c|c|c|c|c|c|}
\hline Name & $\begin{array}{c}S_{8.0}{ }^{\mathrm{a}} \\
(\mathrm{mJy})\end{array}$ & $R$ & $\begin{array}{c}S_{1.4 \mathrm{GHz}}{ }^{\mathrm{b}} \\
(\mathrm{mJy})\end{array}$ & $z_{\text {phot }}{ }^{\mathrm{c}}$ & Optical Type ${ }^{\mathrm{d}}$ \\
\hline SSTXFLS J171106.8+590436 .......... & 1.28 & 19.8 & 0.31 & 0.460 & Galaxy \\
\hline SSTXFLS J171115.2+594906 .......... & 4.53 & 21.0 & 0.12 & $\ldots$ & Stellar \\
\hline SSTXFLS J171133.4+584055 ........... & 1.91 & 18.6 & 0.09 & $\ldots$ & See below \\
\hline SSTXFLS J171147.4+585839.. & 1.56 & 21.0 & 0.57 & $(0.5)$ & Galaxy \\
\hline SSTXFLS J171313.9+603146 .......... & 4.30 & 18.3 & 0.11 & 0.155 & Galaxy \\
\hline SSTXFLS J171324.3+585549 .......... & 1.09 & 20.7 & 0.09 & 0.635 & Galaxy \\
\hline SSTXFLS J171325.1+590531 .......... & 1.19 & 18.3 & 0.16 & 0.105 & Galaxy \\
\hline SSTXFLS J171421.3+602239 ........... & 1.25 & 18.6 & 0.16 & 0.195 & Galaxy \\
\hline SSTXFLS J171430.7+584225 .......... & 1.85 & 19.9 & 0.16 & 0.135 & Galaxy \\
\hline SSTXFLS J171708.6+591341 „........... & 1.32 & 21.1 & $<0.09$ & 0.195 & Galaxy \\
\hline SSTXFLS J171804.6+602705 ........... & 1.05 & 21.7 & $<0.09$ & $(0.6)$ & Galaxy \\
\hline SSTXFLS J171831.6+595317 .......... & 1.10 & 20.6 & 2.22 & 0.295 & Galaxy \\
\hline SSTXFLS J171930.9+594751 _........... & 1.44 & 19.6 & 0.56 & 0.355 & Galaxy \\
\hline SSTXFLS J172050.4+591511 .......... & 3.35 & 21.6 & 1.75 & 0.440 & Galaxy \\
\hline SSTXFLS J172123.1+601214 .......... & 3.40 & 18.8 & 0.26 & 0.355 & Galaxy \\
\hline SSTXFLS J172253.9+582955 ........... & 1.00 & 18.5 & 0.09 & $\ldots$ & Stellar \\
\hline SSTXFLS J172328.4+592947 ........... & 1.48 & 22.2 & 0.31 & $\cdots$ & Stellar \\
\hline SSTXFLS J172432.8+592646 .......... & 3.04 & 15.7 & $<0.09$ & $\ldots$ & Stellar ${ }^{\mathrm{f}}$ \\
\hline SSTXFLS J172458.3+591545 ........... & 1.10 & 20.0 & 0.46 & 0.640 & Galaxy \\
\hline SSTXFLS J172601.8+601100 ........... & 1.43 & 20.3 & 1.26 & $\ldots$ & Stellar \\
\hline
\end{tabular}

a Preliminary flux densities only; errors $\approx 20 \%$.

b Flux densities at $1.4 \mathrm{GHz}$ in the survey of Condon et al. (2003).

c Photometric redshifts $z_{\text {phot }}$; those in brackets are obtained by assuming a galaxy luminosity of $L_{*}$, others are from hyperz using the SDSS ugri magnitudes.

d Optical classification in the $R$-band image.

e This is a stellar object within the isophotes of a spiral disk, and we assume it is a background quasar that happens to be behind a disk galaxy.

${ }^{\mathrm{f}}$ This object may be a star (see text).

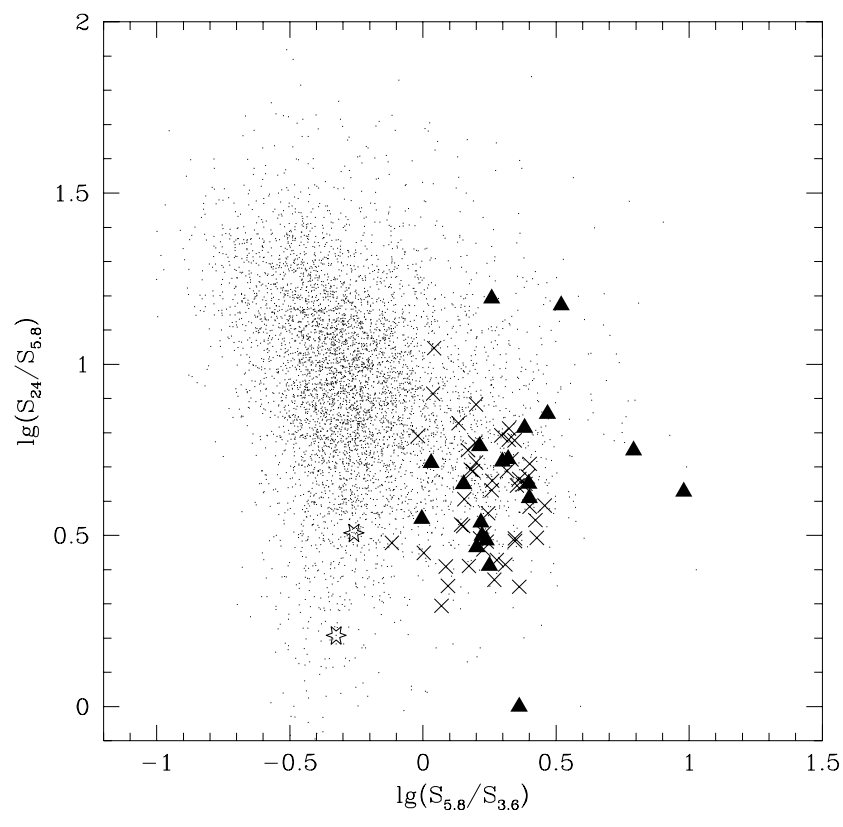

FIG. 2.-IRAC-MIPS $24 \mu \mathrm{m}$ color-color plot. Dots represent objects with "clean" detections in all four IRAC bands matched to the MIPS $24 \mu \mathrm{m}$ catalog of D. Fadda et al. (2004, in preparation), totaling $\sim 6000$ objects. Crosses indicate the colors of SDSS quasars, stars show the SDSS Seyfert 1 galaxies, and triangles mark the $S_{8.0} \geq 1 \mathrm{mJy}$ sample of obscured AGN candidates. plot as the SDSS quasars, consistent with them having SEDs dominated by emission from hot dust around the AGN. Starburst galaxies typically have much redder $S_{24} / S_{5.8}$ colors, because of a lack of high-temperature dust emission from the AGN.

All of our 20 bright, obscured AGN candidates (and all of the SDSS quasars) were identified on the $R$-band images of Fadda et al. (2004; limiting $R \approx 25.5$ ). These identifications allowed us to further refine our selection. Out of the $20 \mathrm{ob}-$ scured AGN candidates, we found that three had the MIR-tooptical color and stellar morphology of normal quasars (one apparently behind a spiral disk), and one candidate was very bright $(R=15.7)$, so it is probably either a peculiar star or a foreground star within $\sim 1^{\prime \prime}$ of a background AGN. Of the remaining 16 objects, 14 are extended in $R$ band and are thus most likely galaxies whose AGNs are completely obscured in the optical. Two are faint in $R$ but are pointlike and so are probably only partly obscured AGN. Figure 3 shows a plot of the ratio of $R$-band flux density $\left(S_{0.65}\right)$ to $S_{8.0}$ plotted against $\log \left(S_{8.0}\right)$ for all 20 objects.

\section{PHOTOMETRIC REDSHIFTS OF THE CANDIDATE AGN POPULATION}

Of the 14 extended (galaxy) detections in $R$ band, 12 are detected on the SDSS DR1 images (which are significantly deeper than the SDSS spectroscopic quasar and galaxy surveys), and we have used their ugri magnitudes to make a rough photometric redshift estimate using hyperz (Bolzonella et al. 2000) with the Bruzual \& Charlot (1993) templates. (We exclude the $z$ band because of the likelihood of contamination by AGN emission, but we believe the other bands should be largely 


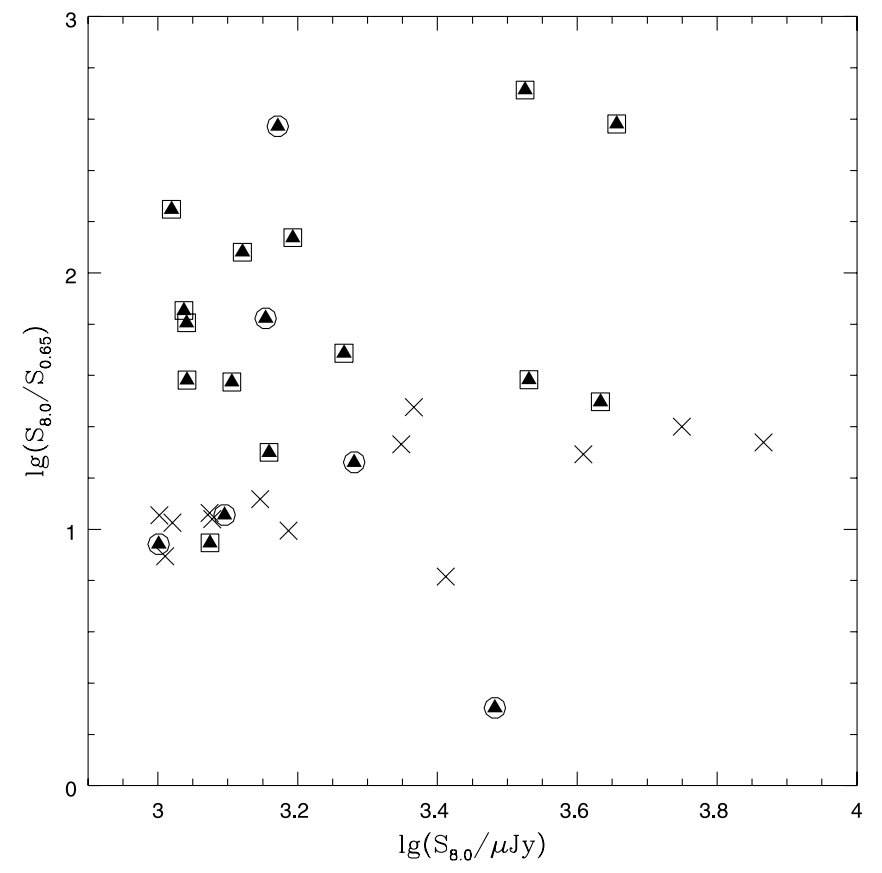

FIG. 3. $-S_{8.0}$ to $R$-band flux ratio as a function of $S_{8.0}$. SDSS quasars are shown as crosses, pointlike identifications of AGN candidates are shown as triangles within circles, and triangles within squares denote galaxy identifications.

free of significant contamination, given the extended appearance of the galaxies in the $R$-band data). The galaxies have a mean luminosity of $\sim L_{*}$, and inspection of the $R$-band image shows that they appear to be mostly early-type galaxies. Our photometric redshifts are only approximate, but the magnitudes of the galaxies are such that few are likely to be at $z \gtrsim 0.7$.

\section{DISCUSSION}

Although we emphasize that the candidate obscured AGNs we have identified from their MIR colors require spectroscopic confirmation as AGNs, our study suggests that about $50 \%$ of the AGNs selected in the MIR at these flux levels may be sufficiently obscured by dust in the optical for them to be missed by the SDSS quasar survey. This excludes AGNs that are not energetically dominant in their host galaxies (which would plot elsewhere on the IRAC color-color diagram) and any AGNs that remain obscured in the MIR.
The median photometric redshift of our candidate obscured AGNs is $\sim 0.36$ (assuming the faint identifications with stellar morphologies lie at high redshift), compared to $\sim 0.69$ for the SDSS quasars with $S_{8.0} \geq 1 \mathrm{mJy}$. There is thus a hint that the obscured AGNs may have lower redshifts and luminosities. Selection effects are unlikely to account for this difference. MIR selection ensures that the AGNs are selected independently of their optical properties. While we might be biased toward objects with more dust close to the AGN, this would not introduce a redshift bias if the fraction of such objects were constant with both luminosity and redshift. A lower median luminosity for the obscured AGNs would, however, be consistent with the "receding torus" model (Lawrence 1991), in which a higher fraction of low-luminosity AGNs are obscured, and might also be consistent with some studies of the X-ray background that find large numbers of low-luminosity, relatively low redshift obscured AGNs (Gilli et al. 2001; Gandhi \& Fabian 2003; Steffen et al. 2003).

If unobscured, our AGNs would be moderately luminous, with $B$-band absolute magnitudes of $M_{B} \approx-22$, on the boundary between Seyfert 1 galaxies and quasars. The most luminous objects would have $M_{B} \sim-23$, which is in the quasar regime and close to the median luminosity of the SDSS sample. Pursuing this work to lower $8 \mu \mathrm{m}$ flux densities, and using spectroscopy to confirm the AGN nature and redshift distribution of our objects, will help us link the infrared to the $\mathrm{X}$-ray studies. This will allow us to derive a consistent story for the nature and evolution of obscured AGNs, and thus for how and when the supermassive black holes in galaxies today accreted most of their mass.

We thank Michael Gregg for assistance with the Lick observations, Susan Ridgway for advice and comments, B. Januzzi and A. Ford for the $R$-band survey, and J. Condon for the Very Large Array survey. We thank the SDSS team for targeting the FLS region in the DR1. This work is based on observations made with the Spitzer Space Telescope, operated by the Jet Propulsion Laboratory (JPL), California Institute of Technology, under NASA contract 1407. Support was provided by NASA through JPL. The SDSS Archive is funded by the Alfred P. Sloan Foundation, the Participating Institutions, NASA, the National Science Foundation, the US Department of Energy, the Japanese Monbukagakusho, and the Max Planck Society.
Bolzonella, M., Miralles, J.-M., \& Pelló, R. 2000, A\&A, 363, 476

Bruzual, G., \& Charlot, S. 1993, ApJ, 405, 538

Condon, J. J., Cotton, W. D., Yin, Q. F., Shupe, D. L., Storrie-Lombardi, L. J., Helou, G., Soifer, B. T., \& Werner, M. W. 2003, AJ, 125, 2411

Cowie, L. L., Barger, A. J., Bautz, M. W., Brandt, W. N., \& Garmire, G. P. 2003, ApJ, 584, L57

Cutri, R. M., Nelson, B. O., Kirkpatrick, J. D., Huchra, J. P., \& Smith, P. S. 2001, in ASP Conf. Ser. 232, The New Era of Wide Field Astronomy, ed. R. Clowes, A. Adamson, \& G. Bromage (San Francisco: ASP), 78

Efstathiou, A., \& Rowan-Robinson, M. 1995, MNRAS, 273, 649

Fadda, D., Jannuzi, B., Ford, A., \& Storrie-Lombardi, L. J. 2004, AJ, 128, 1

Fazio, G. G., et al. 2004, ApJS, 154, 10

Gandhi, P., \& Fabian, A. C. 2003, MNRAS, 339, 1095

Gilli, R., Salvati, M., \& Hasinger, G. 2001, A\&A, 366, 407

Glikman, E., Gregg, M. D., Lacy, M., Helfand, D. J., Becker, R. H., \& White, R. L. 2004, ApJ, 607, 60

Gregg, M. D., Lacy, M., White, R. L., Glikman, E., Helfand, D., Becker, R. H., \& Brotherton, M. S. 2002, ApJ, 563, 133

\section{REFERENCES}

Haas, M., et al. 2004, A\&A, 419, L49

Laurent, O., Mirabel, I. F., Charmandaris, V., Gallais, P., Madden, S. C., Sauvage, M., Vigroux, L., \& Cesarsky, C. 2000, A\&A, 359, 887

Lawrence, A. 1991, MNRAS, 252, 586

Magorrian, J., et al. 1998, AJ, 115, 2285

Norman, C. 2002, ApJ, 571, 218

Pier, E. A., \& Krolik, J. H. 1993, ApJ, 418, 673

Puget, J.-L., \& Léger, A. 1989, ARA\&A, 27, 161

Richards, G. T., et al. 2003, AJ, 126, 1131

Rieke, G. H., et al. 2004, ApJS, 154, 25

Schneider, D. P., et al. 2003, AJ, 126, 2579

Steffen, A. T., Barger, A. J., Cowie, L. L., Mushotzky, R. F., \& Yang, T. 2003, ApJ, 596, L23

Webster, R. L., Francis, P. J., Peterson, B. A., Drinkwater, M. J., Masci, F. J. 1995, Nature, 375, 469

Werner, M., et al. 2004, ApJS, 154, 1

Zakamska, N. L., et al. 2003, AJ, 126, 2125 